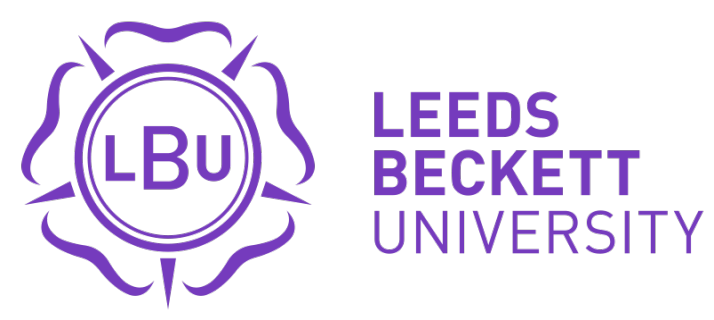

Citation:

Sheikh Akbari, A and Zarachoff, M and Monekosso, D (2019) Single Image Ear Recognition Using Wavelet-Based Multi-Band PCA. 2019 27th European Signal Processing Conference (EUSIPCO). ISSN 2076-1465 DOI: https://doi.org/10.23919/EUSIPCO.2019.8903090

Link to Leeds Beckett Repository record:

https://eprints.leedsbeckett.ac.uk/id/eprint/5959/

Document Version:

Article (Accepted Version)

(C) 2019 IEEE. Personal use of this material is permitted. Permission from IEEE must be obtained for all other uses, in any current or future media, including reprinting/republishing this material for advertising or promotional purposes, creating new collective works, for resale or redistribution to servers or lists, or reuse of any copyrighted component of this work in other works.

The aim of the Leeds Beckett Repository is to provide open access to our research, as required by funder policies and permitted by publishers and copyright law.

The Leeds Beckett repository holds a wide range of publications, each of which has been checked for copyright and the relevant embargo period has been applied by the Research Services team.

We operate on a standard take-down policy. If you are the author or publisher of an output and you would like it removed from the repository, please contact us and we will investigate on a case-by-case basis.

Each thesis in the repository has been cleared where necessary by the author for third party copyright. If you would like a thesis to be removed from the repository or believe there is an issue with copyright, please contact us on openaccess@leedsbeckett.ac.uk and we will investigate on a case-by-case basis. 


\section{Single Image Ear Recognition Using Wavelet-Based Multi-Band PCA}

\author{
Matthew Zarachoff \\ School of Computing, Creative \\ Technologies \& Engineering \\ Leeds Beckett University \\ Leeds, United Kingdom
}

m.zarachoff4868@student.leedsbeckett.ac.uk

\author{
Akbar Sheikh-Akbari \\ School of Computing, Creative \\ Technologies \& Engineering \\ Leeds Beckett University \\ Leeds, United Kingdom
}

\author{
Dorothy Monekosso \\ School of Computing, Creative \\ Technologies \& Engineering \\ Leeds Beckett University \\ Leeds, United Kingdom \\ D.N.Monekosso@leedsbeckett.ac.uk
}

\begin{abstract}
Principal Component Analysis (PCA) has been successfully used for many applications, including ear recognition. This paper presents a 2D Wavelet based Multi-Band PCA (2DWMBPCA) method, inspired by PCA based techniques for multispectral and hyperspectral images, which have shown a significantly higher performance to that of standard PCA. The proposed method performs 2D non-decimated wavelet transform on the input image dividing the image into its subbands. It then splits each resulting subband into a number of bands evenly based on the coefficient values. Standard PCA is then applied on each resulting set of bands to extract the subbands eigenvectors, which are used as features for matching. Experimental results on images of two benchmark ear image datasets show that the proposed 2D-WMBPCA significantly outperforms both the standard PCA method and the eigenfaces method.
\end{abstract}

Index Terms-Ear recognition, principal component analysis, multi-band image creation, non-decimated wavelet transform

\section{INTRODUCTION}

Ear recognition, a field within biometrics, concerns itself with the use of images of the ears to identify individuals. Much like fingerprints, ears are unique to an individual; even identical twins can have distinguishable ears [1]. Researchers have explored this topic extensively over the last two decades, investigating both the feature extraction and comparison of features of ear images [2], [3]. Successful feature extraction techniques in ear recognition include Principal Component Analysis (PCA) [2], [4]-[7], wavelet based [8], and neural network based methods [9]-[11]. Amongst these techniques, PCA has been found to be successful for both feature extraction [4]-[6] and feature reduction to reduce dimensionality of the data [2], [3]. In general, PCA based techniques operate by converting an image into a $1 \mathrm{D}$ vector and concatenating those vectors to form a 2D matrix. While some PCA based ear recognition techniques have been reported in the literature [2], [4], [5], [12], these techniques involve projecting ear images into a common eigenspace. In contrast, the authors previously introduced a single image PCA based technique for ear recognition in [13], inspired by hyperspectral PCA based techniques [14] such as Segmented PCA [15] and Folded PCA [16], which solely use the extracted principal components as features. To the authors' knowledge, no similar techniques that utilize wavelets have been reported in the literature. This has inspired the authors to propose a single image, PCA based method for ear recognition, called 2D Wavelet based MultiBand PCA (2D-WMBPCA). Unlike the aforementioned PCA based methods, the proposed technique does not require the images to be projected into a common eigenspace. Instead, the proposed technique performs a $2 \mathrm{D}$ non-decimated wavelet transform on the input image, dividing the image into its subbands. The resulting subbands are then split evenly into a number of bands according to their their coefficient values. The proposed technique then applies the standard PCA method on each resulting set of bands to extract their principal components as their features that are then used for recognition. Experimental results on the images of two benchmark ear image datasets demonstrate that the proposed 2D-WMBPCA technique greatly outperforms both single image PCA and the eigenfaces technique. The rest of the paper is organized as follows: Section II introduces the proposed 2D-WMBPCA technique, Section III describes the benchmark datasets used, Section IV discusses the experimental results, and finally Section V concludes the paper.

\section{Proposed 2D-WMBPCA Method}

The proposed 2D Wavelet based Multi-Band Principal Component Analysis (2D-WMBPCA) method includes the following five stages: Wavelet Decomposition, Pre-processing, Multiple-image generation, PCA, and Eigenvector matching. Fig. 1 shows the block diagram of the proposed 2D-WMBPCA technique.

\section{A. Wavelet Decomposition}

Let $E$ be the set of all ear images, where each input image $e \in E$ is an 8-bit, grayscale image. The proposed technique performs a 2D non-decimated discrete dyadic wavelet transform on the input image, $e$, using the non-orthogonal wavelet and the fast computation algorithm introduced by Mallat and Hwang in [17], splitting the input image into its four subbands called: Low-Low (LL), Low-High (LH), High-Low (HL), and High-High (HH).

\section{B. Pre-processing}

Coefficients within each subband $s$ are then mapped to the $[0,1]$ domain using $(1)$ : 


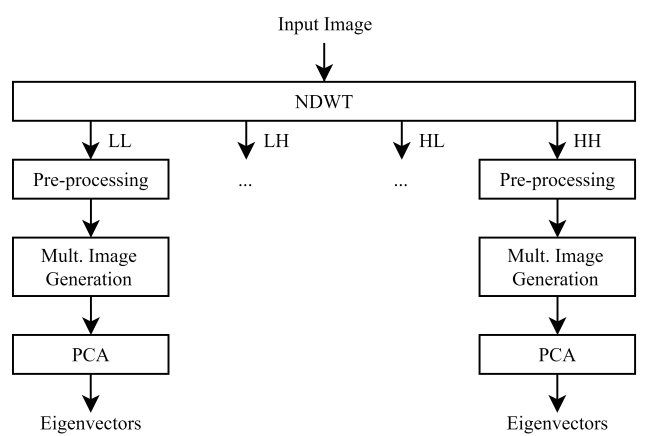

Fig. 1. Block diagram of the proposed 2D-WMBPCA method.

$$
p^{\prime}=\frac{p-\min (s)}{\max (s)-\min (s)}
$$

where $p$ represents an original coefficient in $s$ and $p^{\prime}$ represents the corresponding mapped coefficient.

Histogram equalization is then performed on the resulting subband coefficients to increase their contrast. To do so, the Probability Mass Function (PMF) of the resulting subbands coefficients is first calculated using (2):

$$
P_{X}\left(x_{k}\right)=P\left(X=x_{k}\right) \text { for } k=0,1, \ldots, 255
$$

where $X=x_{1}, x_{2}, \ldots, x_{k}$ represent the subbands' coefficients and $P_{X}\left(x_{k}\right)$ is the probability of coefficients in bin $k$. The resulting PDF is then used to calculate the Cumulative Distribution Function (CDF) of the subband using (3):

$$
C_{X}(k)=P\left(X \leq x_{k}\right) \text { for } k=0,1, \ldots, 255
$$

where $C_{X}(k)$ is the cumulative probability of $X \leq x_{k}$. Finally, all subbands coefficients are mapped to new values using the resulting CDFs. This improves the contrast of the subbands coefficients.

\section{Multiple-Image Generation}

Various methods can be used by proposed 2D Wavelet based Multi-Band PCA (2D-WMBPCA) to generate multiple bands from each input histogram equalized subband. The multiple band generation method used in this research can be formulated as follows:

Assume $s$ is the input subband and $N$ is the number of desired bands to be generated from the subband $s$. The proposed algorithm uses $N-1$ boundaries to split the input subbands coefficients into $N$ target bands according to the coefficient values. Let $B=\left[b_{1}, b_{2}, \ldots, b_{N-1}\right]$ be the boundary values, calculated according to (4):

$$
b_{n}=n / N \text { for } n=1, \ldots,(N-1)
$$

The input subband $s$ coefficients are divided into $\mathrm{N}$ target bands as follows:

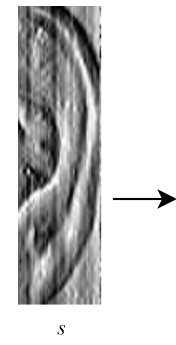

(a)

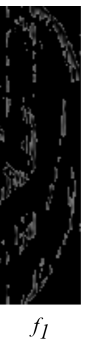

(b)

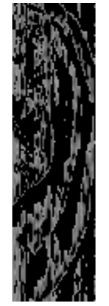

$f_{2}$

(c)

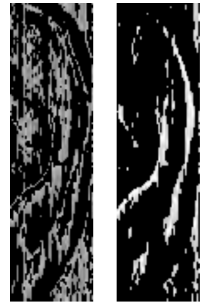

$f_{3}$

(d)

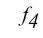

(e)
Fig. 2. An example of multiple image generation: a) Pre-processed input subband $s$ from an image in the IITD II dataset [18], resulting multiple bands b) $f_{1}$ c) $f_{2}$ d) $f_{3}$ e) $f_{4}$. The coefficients for images b) and c) have been multiplied by 1.5 and 1.25 respectively for illustration purposes.

1) Generate $N$ images of the same size of $s$ and set their coefficients to zero. These bands are called:

$F=\left[f_{1}, f_{2}, \ldots, f_{N}\right]$

2) Split the input subbands coefficients into different bands according to their values using the following ranges: $\left[0, b_{1}\right),\left[b_{1}, b_{2}\right), \ldots,\left[b_{N-1}, 1\right]$

It generates $F$ bands from input subband, where these bands, in a sense, form a multispectral image. An illustration of the process to generate four bands can be seen in Fig. 2.

\section{Principal Component Analysis}

Assume $F$ is the set of resulting bands and $F=\left[f_{1}, f_{2}, \ldots, f_{N}\right]$. For each band $f \in F$, a mean adjusted image $f^{\prime}$ is created using (5):

$$
f^{\prime}=f-\bar{f}
$$

where $\bar{f}$ is the mean value of the coefficients in $f$. Every band is then converted to a column wise vector, allowing $F$ to be represented as a two dimensional matrix $W$. PCA is then performed using Singular Value Decomposition (SVD) on matrix $W$, creating the decomposition in (6):

$$
W=U \Sigma V^{T}
$$

where $U$ is a unity matrix and the columns of $V$ are the orthonormal eigenvectors of the covariance matrix of $B$ and $\Sigma$ is a diagonal matrix of their respective eigenvalues. The eigenvectors form a basis for an eigenspace for each set of images $F$. The resulting principal components in $V$ are finally used for matching.

\section{E. Subband Matching}

Let $M=\left[m_{1}, m_{2}, \ldots, m_{N-1}\right]$ be the set of principal components of a query image subband $q$. Furthermore, let $L=\left[l_{1}, l_{2}, \ldots, l_{N-1}\right]$ be the set of principal components of subband $r$, a subband of an image in the image dataset. The set of Euclidean distances $D=\left[d_{1}, d_{2}, \ldots, d_{N-1}\right]$ between $q$ and $r$ can be calculated using (7):

$$
d_{n}=\sqrt{\left(\Sigma_{n}\left(m_{n}-l_{n}\right)^{2}\right)}
$$


The resulting Euclidean distances are summed to produce the total distance between the two subbands. The resulting values for each subband are then summed to produce the total distance between the two images. The best match for query image $q$ in the image database is the image for which the total distance is minimized.

\section{BENCHMARK DATASETS}

This investigation uses two benchmark ear image datasets: The Indian Institute of Technology Delhi II (IITD II) dataset [18] and the University of Science and Technology Beijing II (USTB II) dataset 5 [19]. IITD II dataset consists of 793 images of the right ear of 221 participants. Each participant was photographed between three and six times, with each image being of size $180 \times 50$ pixels and in 8-bit grayscale. For consistency, only the first three images for any individuals are used in this research. The USTB II dataset consists of 308 images of the right ear of 77 participants, each of whom were photographed four times. The photos in this dataset are of size $400 \times 300$ and are also in 8-bit grayscale. While the images in IITD II are tightly cropped, those found in the USTB II dataset contain more of the side of the head. The first image for each participant in USTB II is a frontal image under standard illumination conditions, the second and the third images are each rotated by +30 and -30 degrees, and the fourth image is taken under weak illumination.

\section{EXPERIMENTAL RESULTS}

To assess the performance of the proposed 2D Wavelet based Multi-Band PCA (2D-WMBPCA) technique and compare its performance against standard Principal Component Analysis (PCA) method for ear recognition, the aforementioned two ear image datasets were used. The proposed 2DWMBPCA method using the two boundary selection algorithms described in Section III and the standard PCA method were applied to the images of the two datasets. All of the experiments began by selecting the first image of each subject to serve as a query set and the rest of the images to be a dataset. Given a particular query image, if it is correctly matched with an image in the dataset, it is marked as a Top- 1 image. Similarly, if it is correctly matched with any of the closest five images, it is marked as a Top-5 image. The percentage of Top- 1 and Top-5 images in the dataset are then listed as Top-1 and Top- 5 accuracies. Finally, this process is repeated for the second, third, and in the case of USTB II, fourth images for each individual, with the Top- 1 and Top- 5 accuracies averaged across all trials.

\section{A. Experimental Results for the Standard PCA Method}

To create results for the standard PCA method, the PCA was applied to each original image individually. Their resulting eigenvectors were then compared using Euclidean distance. The results for the application of PCA on both the IITD II and USTB II datasets are presented in Table I. From Table I, it can be seen that the PCA performance on the IITD II image dataset is higher than its performance on the images of the
TABLE I

EXPERIMENTAL RESULTS FOR STANDARD PCA

\begin{tabular}{|c|c|c|}
\hline \multirow{2}{*}{ Dataset } & \multicolumn{2}{|c|}{ Type of Match } \\
\cline { 2 - 3 } & Top-1 & Top-5 \\
\hline IITD II & $36.35 \%$ & $52.94 \%$ \\
\hline USTB II & $17.21 \%$ & $34.09 \%$ \\
\hline
\end{tabular}

TABLE II

EXPERIMENTAL RESULTS FOR THE EIGENFACES PCA METHOD

\begin{tabular}{|c|c|c|}
\hline \multirow{2}{*}{ Dataset } & \multicolumn{2}{|c|}{ Type of Match } \\
\cline { 2 - 3 } & Top-1 & Top-5 \\
\hline IITD II & $89.78 \%$ & $95.64 \%$ \\
\hline USTB II & $24.28 \%$ & $44.93 \%$ \\
\hline
\end{tabular}

USTB II dataset. This can be explained by the fact that some images within the USTB II dataset are rotated.

\section{B. Experimental Results for the Eigenfaces Method}

To generate experimental results for the PCA based eigenfaces method [5], 10\% of images of each ear dataset were used to calculate the eigenvectors. The remaining images were then projected along the resulting eigenvectors to create eigenears, which were compared using the Euclidean distance. The experimental results are tabulated in Table II. From Table II, it can be seen that the eigenfaces method vastly outperforms single image PCA on the images of the IITD II dataset, yet shows a marginal performance increase on the images of the USTB II dataset. This is due to the fact that some of the images of the USTB II dataset are rotated and PCA is sensitive to rotation.

\section{Experimental Results for the Proposed 2D-WMBPCA Method}

The proposed 2D-WMBPCA method was applied to both images of the IITD II and USTB II datasets using two to ten bands of constant size, as discussed in Section III. The number of correct matches was calculated for each set of bands. The results for both the IITD II and USTB II image datasets are tabulated in Table III.

From Table III, it can be seen that the proposed 2DWMBPCA method significantly outperforms the standard PCA method on images of both the IITD II and USTB II datasets. From Table I and II, it is evident that the Top- 1 accuracy of matching has been improved by $57.79 \%$ and $35.69 \%$ on the images of the IITD II dataset using three bins and images of the USTB II dataset using six bins, respectively. From the experiments presented in these two tables, it can be seen that the proposed 2D-WMBPCA achieves its highest performance using fewer bands for the images of the IITD II dataset than for the images of the USTB II dataset. This is likely due to the increased complexity of the images of the USTB II dataset and the fact that the images of the IITD II dataset are more tightly cropped than the images of the USTB II dataset. To compare the performance of the proposed technique with the state of the art PCA and learning based techniques, the Top- 1 experimental results of the proposed $2 \mathrm{D}-\mathrm{CMBPCA}$, single image PCA, 
TABLE III

EXPERIMENTAL RESUlts For THE Proposed TEChNiQue ON THE IITD II DATASET [18]

\begin{tabular}{|c|c|c|}
\hline \multicolumn{2}{|c|}{ Type of Match } & \multirow{2}{*}{ Number of Bands } \\
\hline Top-1 & Top-5 & \\
\hline $90.28 \%$ & $94.64 \%$ & 3 \\
\hline $\mathbf{9 4 . 1 4 \%}$ & $\mathbf{9 7 . 4 9 \%}$ & 3 \\
\hline $92.80 \%$ & $97.32 \%$ & 4 \\
\hline
\end{tabular}

TABLE IV

EXPERIMENTAL RESULTS FOR THE PROPOSED TECHNIQUE ON THE USTB II DATASET [19]

\begin{tabular}{|c|c|c|}
\hline \multicolumn{2}{|c|}{ Type of Match } & \multirow{2}{*}{ Number of Bands } \\
\hline Top-1 & Top-5 & 5 \\
\hline $45.29 \%$ & $68.12 \%$ & $\mathbf{6}$ \\
\hline $\mathbf{5 2 . 9 0 \%}$ & $\mathbf{7 3 . 9 1 \%}$ & 7 \\
\hline $44.93 \%$ & $67.75 \%$ & \\
\hline
\end{tabular}

TABLE V

TOP-1 RESUlts For THE PROPOSED TECHNIQUE AND OTHER PCA AND LEARNING BASED TECHNIQUES

\begin{tabular}{|c|c|c|}
\hline \multirow{2}{*}{ Algorithm } & \multicolumn{2}{|c|}{ Dataset } \\
\cline { 2 - 3 } \multicolumn{2}{|c|}{ PCA based Techniques } \\
\hline II & USTB II \\
\hline Single Image PCA & $36.35 \%$ & $17.21 \%$ \\
\hline Eigenfaces [5] & $89.78 \%$ & $24.28 \%$ \\
\hline 2D-MBPCA [13] & $92.76 \%$ & $\mathbf{5 3 . 9 0 \%}$ \\
\hline Proposed Technique & $\mathbf{9 4 . 1 4 \%}$ & $52.90 \%$ \\
\hline Learning based Techniques \\
\hline BSIF [20] & $97.31 \%$ & - \\
\hline Wavelet and Neural Network [9] & - & $97.50 \%$ \\
\hline
\end{tabular}

eigenfaces [5], 2D-MBPCA [13], BSIF [20], and wavelet and neural network based [9] techniques are tabulated in Table V. From Table V, it is apparent that the proposed 2D-WMBPCA technique significantly outperforms the single image PCA and the eigenfaces method and gives competitive results for the images of the IITD II dataset when compared with learning based techniques. Furthermore, it shows a slight improvement to the results of the 2D-MBPCA method on the IITD II dataset. However, due to the use of PCA, a rotation variant transform, the performance of the PCA based techniques is much below the state of the art learning based methods on the images of the USTB II dataset.

\section{CONCLUSION}

In this paper, a non-decimated wavelet and PCA based ear recognition algorithm, called 2D Wavelet based Multi-Band PCA (2D-WMBPCA), was presented. Experimental results on the images of two benchmark ear image datasets show that the proposed 2D-WMBPCA technique significantly outperforms both the standard PCA and the eigenfaces methods. Furthermore, the 2D-WMBPCA shows improved accuracy when compared with the previously published 2D-MBPCA algorithm on one of two benchmark datasets, suggesting that the features extracted by the wavelet transform play a crucial role in PCA based ear recognition techniques.

\section{REFERENCES}

[1] H. Nejati, L. Zhang, T. Sim, E. Martinez-Marroquin, and G. Dong, "Wonder ears: Identification of identical twins from ear images," in Proceedings of the 21st International Conference on Pattern Recognition (ICPR2012), Nov. 2012, pp. 1201-1204.

[2] Ž. Emeršič, V. Štruc, and P. Peer, "Ear recognition: More than a survey," Neurocomputing, vol. 255, pp. 26-39, Sep. 2017. [Online]. Available: http://linkinghub.elsevier.com/retrieve/pii/S092523121730543X

[3] A. Pflug and C. Busch, "Ear biometrics: a survey of detection, feature extraction and recognition methods," IET Biometrics, vol. 1, no. 2, pp. 114-129, Jun. 2012.

[4] B. Victor, K. Bowyer, and S. Sarkar, "An evaluation of face and ear biometrics," in Object recognition supported by user interaction for service robots, vol. 1, 2002, pp. 429-432 vol.1.

[5] K. Chang, K. W. Bowyer, S. Sarkar, and B. Victor, "Comparison and combination of ear and face images in appearance-based biometrics," IEEE Transactions on Pattern Analysis and Machine Intelligence, vol. 25 , no. 9 , pp. 1160-1165, Sep. 2003.

[6] D. Querencias-Uceta, B. Ríos-Sánchez, and C. Sánchez-Ávila, "Principal component analysis for ear-based biometric verification," in 2017 International Carnahan Conference on Security Technology (ICCST), Oct. 2017, pp. 1-6.

[7] M. A. Turk and A. P. Pentland, "Face recognition using eigenfaces," in 1991 IEEE Computer Society Conference on Computer Vision and Pattern Recognition Proceedings, Jun. 1991, pp. 586-591.

[8] M. S. Nosrati, K. Faez, and F. Faradji, "Using 2d wavelet and principal component analysis for personal identification based On $2 \mathrm{~d}$ ear structure," in 2007 International Conference on Intelligent and Advanced Systems, Nov. 2007, pp. 616-620.

[9] L. Gutierrez, P. Melin, and M. Lopez, "Modular neural network integrator for human recognition from ear images," in The 2010 International Joint Conference on Neural Networks (IJCNN), Jul. 2010, pp. 1-5.

[10] F. I. Eyiokur, D. Yaman, and H. K. Ekenel, "Domain adaptation for ear recognition using deep convolutional neural networks," IET Biometrics, vol. 7, no. 3, pp. 199-206, 2018.

[11] S. Dodge, J. Mounsef, and L. Karam, "Unconstrained ear recognition using deep neural networks," IET Biometrics, vol. 7, no. 3, pp. 207-214, 2018.

[12] J. Yang, D. Zhang, A. F. Frangi, and J.-y. Yang, "Two-dimensional PCA: a new approach to appearance-based face representation and recognition," IEEE Transactions on Pattern Analysis and Machine Intelligence, vol. 26, no. 1, pp. 131-137, Jan. 2004.

[13] M. Zarachoff, A. Sheikh-Akbari, and D. Monekosso, "2d Multi-Band PCA and its Application for Ear Recognition," in 2018 IEEE International Conference on Imaging Systems and Techniques (IST), Oct. 2018, pp. $1-5$.

[14] J. C. Harsanyi and C. I. Chang, "Hyperspectral image classification and dimensionality reduction: an orthogonal subspace projection approach," IEEE Transactions on Geoscience and Remote Sensing, vol. 32, no. 4, pp. 779-785, Jul. 1994.

[15] X. Jia and J. A. Richards, "Segmented principal components transformation for efficient hyperspectral remote-sensing image display and classification," IEEE Transactions on Geoscience and Remote Sensing, vol. 37, no. 1, pp. 538-542, Jan. 1999.

[16] J. Zabalza, J. Ren, M. Yang, Y. Zhang, J. Wang, S. Marshall, and J. Han, "Novel Folded-PCA for improved feature extraction and data reduction with hyperspectral imaging and SAR in remote sensing," ISPRS Journal of Photogrammetry and Remote Sensing, vol. 93, pp. 112-122, Jul. 2014. [Online]. Available: http://www.sciencedirect.com/science/article/pii/S0924271614000938

[17] S. Mallat and W. L. Hwang, "Singularity detection and processing with wavelets," IEEE Transactions on Information Theory, vol. 38, no. 2, pp. 617-643, Mar. 1992.

[18] "IIT Delhi Ear Database." [Online]. Available: http://www4.comp.polyu.edu.hk/csajaykr/IITD/Database_Ear.htm

[19] "Ear Recoginition Laboratory at USTB." [Online]. Available: http://www1.ustb.edu.cn/resb/en/index.htm

[20] A. Benzaoui, N. Hezil, and A. Boukrouche, "Identity recognition based on the external shape of the human ear," in 2015 International Conference on Applied Research in Computer Science and Engineering (ICAR), Oct. 2015, pp. 1-5. 